\title{
Retraction Note: Hybrid recurrent Laguerre-orthogonal-polynomials neural network control with modified particle swarm optimization application for V-belt continuously variable transmission system
}

\author{
Chih-Hong Lin ${ }^{1}$
}

Published online: 28 January 2021

(C) Springer-Verlag London Ltd., part of Springer Nature 2021

Retraction Note: Neural Comput \& Applic (2017) 28:245-264 https://doi.org/10.1007/s00521-015-2053-2

The Editor-in-Chief has retracted this article [1] because it significantly overlaps with a number of articles including those that were under consideration at the same time [2-4] and previously published [5-7].

Author Chih-Hong Lin agrees to this retraction.

\section{References}

1. Lin CH (2017) Hybrid recurrent Laguerre-orthogonal-polynomials neural network control with modified particle swarm optimization application for V-belt continuously variable transmission system. Neural Comput Appl 28(2):245-264

2. Lin CH (2015) Hybrid recurrent Laguerre-orthogonal-polynomial $\mathrm{NN}$ control system applied in V-belt continuously variable transmission system using particle swarm optimization. Math Problems Eng 2015
3. Lin CH (2015) Application of hybrid recurrent Laguerre-orthogonal-polynomial $\mathrm{NN}$ control in V-belt continuously variable transmission system using modified particle swarm optimization. J Mech Sci Technol 29(9):3933-3952

4. Lin CH (2016) Novel application of continuously variable transmission system using composite recurrent Laguerre orthogonal polynomials modified PSO NN control system. ISA Trans 64:405-417

5. Lin CH (2015) Application of V-belt continuously variable transmission system using hybrid recurrent Laguerre orthogonal polynomials neural network control system and modified particle swarm optimization. J Comput Nonlinear Dyn 10(5)

6. Lin CH (2015) Composite recurrent Laguerre orthogonal polynomials neural network dynamic control for continuously variable transmission system using altered particle swarm optimization. Nonlinear Dyn 81(3):1219-1245

7. Lin $\mathrm{CH}$ (2016) An intelligent dynamic control of continuously variable transmission system using modified particle swarm optimization. Proc Inst Mech Eng Part C J Mech Eng Sci 230(13):2181-2207

Publisher's Note Springer Nature remains neutral with regard to jurisdictional claims in published maps and institutional affiliations.

The original article can be found online at https:// doi.org/10.1007/s00521-015-2053-2.

Chih-Hong Lin

jhlin@nuu.edu.tw

1 Department of Electrical Engineering, National United

University, No. 1, Lienda, Kung-Jing Li,

Maioli City 36003, Miaoli County, Taiwan 